\title{
Frequency Aliasing-Based Spatial-Wavenumber Filter for Online Damage Monitoring
}

\author{
Bin Liu $\left(\mathbb{D},{ }^{1,2}\right.$ Tingzhang Liu $\mathbb{D}^{2},{ }^{2}$ Jianfei Zhao, ${ }^{2}$ and Dan Hang ${ }^{1}$ \\ ${ }^{1}$ Department of Quartermaster and Aviation POL, Air Force Logistics College, Xuzhou 221000, China \\ ${ }^{2}$ School of Mechatronic Engineering and Automation, Shanghai University, Shanghai 200444, China \\ Correspondence should be addressed to Tingzhang Liu; liutzhcom@oa.shu.edu.cn
}

Received 21 April 2020; Revised 1 June 2020; Accepted 11 June 2020; Published 3 August 2020

Academic Editor: Anil Kumar

Copyright $\odot 2020$ Bin Liu et al. This is an open access article distributed under the Creative Commons Attribution License, which permits unrestricted use, distribution, and reproduction in any medium, provided the original work is properly cited.

\begin{abstract}
The spatial-wavenumber filter method can extract the specific mode of the Lamb wave, thereby distinguishing the incident wave and the damage reflection wave. This method has been widely studied for damage imaging. However, the diameter of piezoelectric transducer (PZT) sensor limits the spatial sampling wavenumber of the linear PZT sensor array, which limits the application of this method because of the Nyquist-Shannon sampling theorem. Therefore, the wavenumber filtering range of spatial-wavenumber filter should be less than half of the spatial sampling wavenumber. In this paper, a frequency aliasing based spatialwavenumber filter for online damage monitoring is proposed. In this method, the wavenumber filtering range is extended to the spatial sampling wavenumber, and two wavenumber results will be calculated as for the frequency aliasing. Subsequently, the wavenumber of the received Lamb wave signal can be obtained according to the average arrival time difference between the two adjacent sensors in the linear PZT sensor array. Finally, the damage is localized using the spatial-wavenumber filter and cruciform PZT sensor array. This method was validated on an epoxy laminate plate. The maximum damage localization errors are less than $2 \mathrm{~cm}$. It is indicated that this method can extend the spatial-wavenumber filtering range to the spatial sampling wavenumber and the application of spatial-wavenumber filter-based online damage monitoring.
\end{abstract}

\section{Introduction}

According to the concept of smart material structure, structural health monitoring (SHM) technology involves the application of embedded sensor networks to obtain information related to structural health online. The characteristic parameters of the signal related to structural health are extracted by an advanced signal processing algorithm. Thus, we can determine whether the structure is damaged, localize the damage, analyze the degree of damage, and predict the failure form and remaining life of the damaged structure. Therefore, SHM technology can be used to prevent the occurrence of major accidents, improve safety of the structure, and reduce economic losses $[1,2]$. As a type of elastic stress wave, the Lamb wave is widely applied in damage identification of composite structures because of its long propagation distance, small energy attenuation, and sensitive response to damage. Therefore, SHM technology based on the Lamb wave has been widely studied and is one of the most promising SHM technologies. In such applications, the Lamb wave is excited and collected by a low-cost piezoelectric transducer (PZT) sensor [3-5].

In the existing SHM technologies, the damage imaging method which has high signal-to-noise ratio can visually indicate damage location and size. Examples of these imaging techniques include the delay-and-sum method [6-9], time reversal method [10-13], probabilistic diagnostic algorithm [14-17], phased array method [18-21], multisignal classification method [22-26], and spatial-wavenumber filter method [27-35]. Among them, the spatial-wavenumber filter method, which has been extensively studied, can extract the specific mode of the Lamb wave, distinguish the incident wave and damage scattering wave, and reduce the overlap of Lamb wave signals [33-35]. Purekar and Pines [27] introduced the spatial-wavenumber filter into the Lamb wave and linear PZT sensor array based damage imaging first 
and performed damage monitoring of large aluminum plates based on the wavenumber of the Lamb wave signal acquired by structural mechanical modeling. Wang et al. [28] improved the spatial-wavenumber filter independent of the structural material parameters using the envelope of Lamb wave damage scattering signal extracted by the Hilbert transform. Qiu et al. [29, 30] studied a scanning spatialwavenumber filter for damage and impact localization of composite structures without using the structural model. Ren et al. [31, 32] extended the model-independent spatialwavenumber filter to multidamage and impact monitoring using eigenvalue decomposition and wavenumber searching.

In previous spatial-wavenumber filter damage imaging research, the Lamb wave was collected using linear PZT sensor array. According to the Nyquist-Shannon sampling theorem, the frequency of the Lamb wave must be less than half of the sampling frequency. Similarly, the wavenumber of the Lamb wave also must be less than half of the spatial sampling wavenumber. However, the diameter of PZT sensor which is difficult to increase limits the spatial sampling wavenumber. Thus, it will limit the application of spatial-wavenumber filter based online damage monitoring. In this study, a frequency aliasing based spatial-wavenumber filter for online damage monitoring is proposed, which extends the spatial-wavenumber filtering range to the spatial sampling wavenumber. The basic principle of the spatialwavenumber filter is introduced in Section 2. Then, the damage is localized using the spatial-wavenumber filter and cruciform PZT sensor array that is described in Section 3. The proposed spatial-wavenumber filter is validated on an epoxy laminate plate in Section 4. Finally, the conclusions are stated in Section 5.

\section{Spatial-Wavenumber Filter}

2.1. Theoretic Foundation. Figure 1 shows a linear PZT sensor array placed on a structure. There are $M$ PZT sensors in the linear PZT sensor array and are numbered $m=1, \ldots$, $M$. The spatial sampling interval is $\Delta x$, which is also equivalent to the distance between the centers of two adjacent PZT sensors in the linear PZT sensor array. A Cartesian coordinate was built on the structure. The original point was set at the center point of the linear PZT sensor array, and the $X$-axis was set along the linear PZT sensor array.

As illustrated in Figure 1, the acoustic source located at $\left(\theta_{a}, L_{a}\right)$ excites the Lamb wave signal in the structure. The acoustic source is in the far-field area of the linear PZT sensor array. The propagation of the Lamb wave in the structure can be expressed using [36]

$$
f(x, t)=A \cdot e^{i \cdot\left(\omega_{a} t-k_{a} x+\varphi_{0}\right)},
$$

where $x$ and $t$ represent the propagation distance and time of the Lamb wave, respectively; $A$ denotes the amplitude term of the Lamb wave; $\omega_{a}$ and $k_{a}$ are the central frequency and wavenumber of the Lamb wave; $\varphi_{0}$ is the initial phase of the Lamb wave.

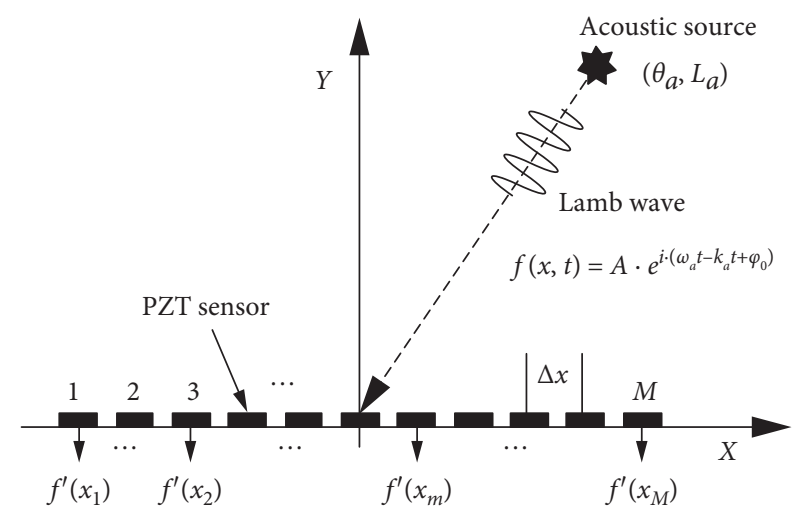

FIGURE 1: Diagram of the Lamb wave spatial sampling.

The wavenumber response can be obtained by Fourier Transform of the spatial response shown in (1) and (2). Then, the wavenumber domain of the Lamb wave can be obtained as follows:

$$
\begin{aligned}
F(k) & =\int_{-\infty}^{\infty} f(x, t) \cdot e^{-i k x} \mathrm{~d} x, \\
& =\int_{-\infty}^{\infty} B \cdot e^{i \cdot k_{a} \cdot x} \cdot e^{-i k x} \mathrm{~d} x, \\
& =B \cdot 2 \pi \cdot \delta\left(k-k_{a}\right),
\end{aligned}
$$

where $\delta$ is the Dirac function and

$$
B=A \cdot e^{i \cdot\left(\omega_{a} t+\varphi_{0}\right)} .
$$

Using the linear PZT sensor array, the discrete spatial sampling signal $f^{\prime}(x, t)$ can be obtained by spatial sampling of Lamb wave with a spatial sampling interval of $\Delta x$, as shown in (4). In other words, the spatial sampling signal $f^{\prime}(x, t)$ is the product of the Lamb wave propagating continuously and the periodic impact signal $p(x)$ :

$$
\begin{aligned}
f^{\prime}(x, t) & =f(x, t) \cdot p(x), \\
& =B \cdot e^{i \cdot k_{a} \cdot x} \cdot \sum_{n=-\infty}^{\infty} \delta(x-n \cdot \Delta x), \\
& =B \cdot \sum_{n=-\infty}^{\infty} e^{i \cdot k_{a} \cdot n \cdot \Delta x} \cdot \delta(x-n \cdot \Delta x),
\end{aligned}
$$

where $n$ is an integer and

$$
p(x)=\sum_{n=-\infty}^{\infty} \delta(x-n \cdot \Delta x) .
$$

Using the Fourier Transform, the wavenumber response $P(x)$ of the periodic impact signal $p(x)$ can be obtained, as shown in

$$
\begin{aligned}
P(k) & =\int_{-\infty}^{\infty} p(x) \cdot e^{-i k x} \mathrm{~d} x, \\
& =\frac{2 \pi}{\Delta x} \sum_{n=-\infty}^{\infty} \delta\left(k-n \cdot k_{s}\right),
\end{aligned}
$$

where $k_{s}=2 \pi / \Delta x$ is the spatial sampling wavenumber of the linear PZT sensor array. 
According to (2) and (6), the wavenumber response of the discrete spatial sampling signal $f^{\prime}(x, t)$ is shown in

$$
\begin{aligned}
F^{\prime}(k, t) & =\int_{-\infty}^{\infty} f(x, t) \cdot p(x) \cdot e^{-i k x} \mathrm{~d} x, \\
& =\frac{1}{2 \pi} \cdot S(k) \otimes P(k), \\
& =\frac{B \cdot 2 \pi}{\Delta x} \sum_{n=-\infty}^{\infty} \delta\left(k-k_{a}-n \cdot k_{s}\right) .
\end{aligned}
$$

Equation (7) shows that in the range of wavenumber domain $\left(-k_{s}, k_{s}\right)$ we have the following:

(1) $k_{a} \geq 0$ :

(a) If $k_{s} \geq 2 k_{a}$, the wavenumber results of the discrete spatial sampling signal $f^{\prime}(x, t)$ are $k_{a}$ and $\left(k_{a}-k_{s}\right)$, and $\left(k_{a}-k_{s}\right) \in\left(-k_{s},-k_{a}\right]$, which is negative

(b) If $k_{a} \leq k_{s}<2 k_{a}$, the wavenumber results of the discrete spatial sampling signal $f^{\prime}(x, t)$ are $k_{a}$ and $\left(k_{a}-k_{s}\right)$, and $\left(k_{a}-k_{s}\right) \in\left(-k_{s}, 0\right]$, which is negative

(c) If $0.5 k_{a} \leq k_{s}<k_{a}$, the wavenumber results of the discrete spatial sampling signal $f^{\prime}(x, t)$ are $\left(k_{a}-k_{s}\right)$ and $\left(k_{a}-2 k_{s}\right) ;\left(k_{a}-k_{s}\right) \in\left(0, k_{s}\right]$, which is positive, and $\left(k_{a}-2 k_{s}\right) \in\left(-k_{s}, 0\right]$, which is negative

(2) $k_{a}<0$ :

(a) If $k_{s} \geq 2\left|k_{a}\right|$, the wavenumber results of the discrete spatial sampling signal $f^{\prime}(x, t)$ are $k_{a}$ and $\left(k_{a}+k_{s}\right)$, and $\left(k_{a}+k_{s}\right) \in\left[k_{a}, k_{s}\right)$, which is positive

(b) If $\left|k_{a} \leq k_{s}<2\right| k_{a} \mid$, the wavenumber results of the discrete spatial sampling signal $f^{\prime}(x, t)$ are ka and $\left(k_{a}+k_{s}\right)$, and $\left(k_{a}+k_{s}\right) \in\left[0, k_{s}\right)$, which is positive

(c) If $0.5\left|k_{a}\right| \leq k_{s}<\left|k_{a}\right|$, the wavenumber results of the discrete spatial sampling signal $f^{\prime}(x, t)$ are $\left(k_{a}+k_{s}\right)$ and $\left(k_{a}+2 k_{s}\right) ;\left(k_{a}+k_{s}\right) \in\left(-k_{s}, 0\right]$, which is negative, and $\left(k_{a}+2 k_{s}\right) \in\left(0, k_{s}\right]$, which is positive

As discussed above, the spatial sampling wavenumber $k_{s}$ should be greater than twice that of the Lamb wave according to the Nyquist-Shannon sampling theorem, as shown in (8). Furthermore, there is only one calculated wavenumber result $k_{a}$ in the range of $\left(-0.5 k_{s}, 0.5 k_{s}\right)$ which is the wavenumber $k_{a}$ of the Lamb wave in the previous research:

$$
k_{s}>2 k_{a} .
$$

Therefore, if $k_{a} \neq 0$, there will be two calculated wavenumber results $k_{a}$ and $\left(\left(k_{a}-k_{s}\right)\right.$ or $\left.\left(k_{a}+k_{s}\right)\right)$ in the range of $\left(-k_{s}, k_{s}\right)$ and $k_{s} \geq 2\left|k_{a}\right|$ or $\left|k_{a}\right| \leq k_{s}<2\left|k_{a}\right|$. The signs of the two calculated wavenumber results are opposite. Thus, the wavenumber $k_{a}$ of the Lamb wave can be obtained when the sign of the wavenumber $k_{a}$ can be determined.

2.2. Principle of the Method. As shown in Figure 1, the spatial sampling wavenumber of the linear PZT sensor array exceeds that of the Lamb wave, $k_{s}>\left|k_{a}\right|$. In addition, the received Lamb wave signals collected by the linear PZT sensor array can be expressed as shown in

$$
\begin{aligned}
f^{\prime}\left(x_{m}, t\right) & =B \cdot e^{-i \cdot k_{a} \cdot\left|\vec{L}_{a}-\vec{X}_{m}\right|} \approx B \cdot e^{-i \cdot k_{a} \cdot L_{a}} \cdot e^{i \cdot k_{a} \cdot \cos \theta_{a} \cdot x_{m}} \\
& =C \cdot e^{i \cdot k_{a} \cdot \cos \theta_{a} \cdot x_{m}} \quad(m=1, \ldots, M),
\end{aligned}
$$

where $\vec{L}_{a}$ is the vector of the distance $L_{a}$ from the position of the acoustic source to the origin point; $\vec{X}_{m}$ is the vector of the $X$-axis coordinate $x_{m}$ of the No.m PZT sensor:

$$
C=B \cdot e^{-i \cdot k_{a} \cdot L_{a}}
$$

According to (7), the received Lamb wave signal shown in (9) is transformed to the wavenumber response, as shown in

$$
F^{\prime \prime}(k, t)=\frac{C \cdot 2 \pi}{\Delta x} \sum_{n=-\infty}^{\infty} \delta\left(k-k_{a} \cdot \cos \theta_{a}-n \cdot k_{s}\right) .
$$

A spatial-wavenumber filter is designed for the received Lamb wave signal, as shown in (12). Using Fourier Transform, the wavenumber response of the spatial-wavenumber filter can be obtained, as shown in

$$
\begin{aligned}
\phi_{k^{\prime}}(x) & =e^{i \cdot k^{\prime} \cdot x}, \\
\Phi(k) & =\int_{-\infty}^{\infty} \phi_{k^{\prime}}(x) \cdot e^{-i k x} \mathrm{~d} x, \\
& =\int_{-\infty}^{\infty} e^{i \cdot k^{\prime} \cdot x} \cdot e^{-i k x} \mathrm{~d} x, \\
& =2 \pi \cdot \delta\left(k-k^{\prime}\right),
\end{aligned}
$$

where $k^{\prime}$ is the pass-through wavenumber of the spatialwavenumber filter.

Equation (13) shows that the spatial-wavenumber filter can selectively pass through the signal with the wavenumber that is $k=k^{\prime}$ and reject the signal with the other wavenumbers $k \neq k^{\prime}$.

Next, the designed spatial-wavenumber filter is applied to the received Lamb wave signal when the wavenumber filtering range is $\left(-k_{s}, k_{s}\right)$, as shown in (14). The filtered wavenumber response of the received Lamb wave signal can be expressed as

$$
h_{k^{\prime}}\left(x_{m}, t\right)=f^{\prime}\left(x_{m}, t\right) \otimes \phi_{k^{\prime}}\left(x_{m}\right)
$$

$$
\begin{aligned}
H(k, t)= & 2 \pi \cdot \delta\left(k-k^{\prime}\right) \\
& \cdot \frac{C \cdot 2 \pi}{\Delta x} \sum_{n=-\infty}^{\infty} \delta\left(k-k_{a} \cdot \cos \theta_{a}-n \cdot k_{s}\right), \\
= & \frac{4 \pi^{2} C}{\Delta x} \cdot \delta\left(k-k^{\prime}\right) \cdot \sum_{n=-\infty}^{\infty} \delta\left(k-k_{a} \cdot \cos \theta_{a}-n \cdot k_{s}\right) .
\end{aligned}
$$

Finally, the spatial-wavenumber filtered synthesis signal of the linear PZT sensor array can be obtained using

$$
h^{\prime}\left(k^{\prime}, t\right)=\sum_{m=1}^{M}\left|f^{\prime}\left(x_{m}, t\right) \otimes \phi_{k^{\prime}}\left(x_{m}\right)\right| .
$$


According to (15), the amplitude value of the spatialwavenumber filtered synthesis signal is small when $k^{\prime} \neq\left(k_{a} \cdot \cos \theta_{a}-n \cdot k_{s}\right)$. When $k^{\prime}=\left(k_{a} \cdot \cos \theta_{a}-n \cdot k_{s}\right)$, the amplitude value will be maximum. Therefore, by applying the designed spatial-wavenumber filter to the Lamb wave received signal with the wavenumber filtering range from $-k_{s}$ to $+k_{s}$, the $\left(k_{a} \cdot \cos \theta_{a}-n \cdot k_{s}\right)$ value corresponding to the maximum value of spatial-wavenumber filtered synthesis signal can be obtained.

According to the analysis in the previous section, there are two positive and negative wavenumber results in the range of $\left(-k_{s}, k_{s}\right)$ when $k_{a} \cdot \cos \theta_{a} \neq 0$. If $k_{a} \cdot \cos \theta_{a}>0$, $\left(k_{a} \cdot \cos \theta_{a}-k_{s}\right) \quad$ will be negative. If $k_{a} \cdot \cos \theta_{a}<0$, $\left(k_{a} \cdot \cos \theta_{a}+k_{s}\right)$ will be negative.

There will be only one value of $0 \mathrm{rad} / \mathrm{m}$ which can be obtained when $k_{a} \cdot \cos \theta_{a}=0$, which is the wavenumber of the received Lamb wave signal collected by the linear PZT sensor array.

In addition, Figure 1 shows that if the damage is at the right side of the $Y$-axis, that is, the positive half axis of the $X$-axis, the arrival time of the received Lamb wave signal collected by the No.M PZT sensor will be earlier than that of the signal collected by the No.1 PZT sensor and $k_{a} \cdot \cos \theta_{a}>0$. Otherwise, the arrival time of the Lamb wave received signal collected by the No.M PZT sensor will be later than that of signal collected by the No.1 PZT sensor and $k_{a} \cdot \cos \theta_{a}<0$, when the damage is at the left side of the $Y$-axis. Therefore, when the spatial-wavenumber filtering result has two values, the wavenumber of the received Lamb wave signal can be finally determined by comparing the arrival times of the received Lamb wave signals collected by the No.M and No.1 PZT sensors in the linear PZT sensor array.

In practical application, the spatial-wavenumber filtering result and the calculated arrival time considerably fluctuate because of various factors which can easily cause misjudgment. Therefore, the average arrival time difference $t_{a}$ between two adjacent sensors can be calculated using

$$
t_{a}=\frac{\sum_{m=1}^{M-1}\left(t_{(m+1)}-t_{m}\right)}{M-1},
$$

where $t_{m}$ is the arrival time of the received Lamb wave signal collected by the No.m PZT sensor and $t_{(m+1)}$ is the arrival time of the received Lamb wave signal collected by the No. $(m+1)$ PZT sensor.

Equation (17) shows that the arrival time of the received Lamb wave signals collected by the No.M PZT sensor will be later than that of the signal collected by the No.1 PZT sensor if $t_{a}>0$, which means $k_{a} \cdot \cos \theta_{a}<0$. Otherwise, if $t_{a}<0$, the arrival time of the received Lamb wave signals collected by the No.M PZT sensor will be earlier than that of signal collected by the No.1 PZT sensor and $k_{a} \cdot \cos \theta_{a}>0$.

Finally, the wavenumber of the received Lamb wave signal can be obtained.

Using the linear PZT sensor array, the received Lamb wave signals can be collected for a certain length of time. Then, a wavenumber-time image can be obtained by spatialwavenumber filtering of the received Lamb wave signals at each time, as shown in Figure 2. In Figure 2, the wavenumber and time corresponding to the image point of the highest pixel value can be judged to be the spatial-wavenumber filtering result $\left(k_{a} \cdot \cos \theta_{a}-n \cdot k_{s}\right)$ and the arrival time $t_{R}$ of the received Lamb wave signal. Therefore, the wavenumber $k_{a} \cdot \cos \theta_{a}$ and the arrival time $t_{R}$ of the Lamb wave received signal can be obtained simultaneously by the spatial-wavenumber filter.

\section{Damage Localization}

There is a cruciform PZT sensor array in the structure which is constructed by two linear PZT sensor arrays, as shown in Figure 3. The two linear PZT sensor arrays of the cruciform PZT sensor array are labeled as No.I and No.II. A Cartesian coordinate is built on the structure. The original point is set at the cross point of the cruciform PZT sensor array, and the $X$ - and $Y$-axis are set along the No.I and No.II PZT sensor arrays. The Lamb wave is excited from the center point and propagation in the structure. If there is damage in the structure, it will scatter the incident Lamb wave [37]. The damage scattering signal can be collected by the cruciform PZT sensor array for a certain length of time.

The values of $k_{a-\mathrm{I}}=k_{a} \cos \theta_{a}$ and $t_{R-\mathrm{I}}$ can be obtained by spatial-wavenumber filtering of the damage scattering signal collected by No.I linear PZT sensor array, as shown in Figure 3 . In addition, $k_{a-\mathrm{II}}=k_{a} \cos \left(90^{\circ}-\theta_{a}\right)$ and $t_{R-\mathrm{II}}$ can be obtained by spatial-wavenumber filtering of the damage scattering signal collected by No.II linear PZT sensor array. Thus, the $X$-axis and $Y$-axis projection wavenumbers of the damage scattering signals all can be calculated using the spatial-wavenumber filter and cruciform PZT sensor array. Then, the angle $\theta_{a}$ of damage can be calculated using (18). Furthermore, the distance $L_{a}$ of damage can be calculated using the following equation. Finally, the damage position $\left(\theta_{a}, L_{a}\right)$ is localized:

$$
\theta_{a}= \begin{cases}\arctan \left(\frac{k_{a-\mathrm{II}}}{k_{a-\mathrm{I}}}\right), & \left(k_{a-\mathrm{I}}>0, k_{a-\mathrm{II}} \geq 0\right), \\ 90^{\circ}, & \left(k_{a-\mathrm{I}}=0, k_{a-\mathrm{II}}>0\right), \\ 180^{\circ}+\arctan \left(\frac{k_{a-\mathrm{II}}}{k_{a-\mathrm{I}}}\right), & \left(k_{a-\mathrm{I}}<0\right), \\ 270^{\circ}, & \left(k_{a-\mathrm{I}}=0, k_{a-\mathrm{II}}<0\right), \\ 360^{\circ}+\arctan \left(\frac{k_{a-\mathrm{II}}}{k_{a-\mathrm{I}}}\right), & \left(k_{a-\mathrm{I}}<0, k_{a-\mathrm{II}}>0\right),\end{cases}
$$

$$
L_{a}=\frac{1}{2} \cdot c_{g} \cdot\left(\frac{t_{R-\mathrm{I}}+t_{R-\mathrm{II}}}{2}-t_{e}\right)
$$

where $c_{g}$ is the Lamb wave group velocity and $t_{e}$ is the Lamb wave start time. 


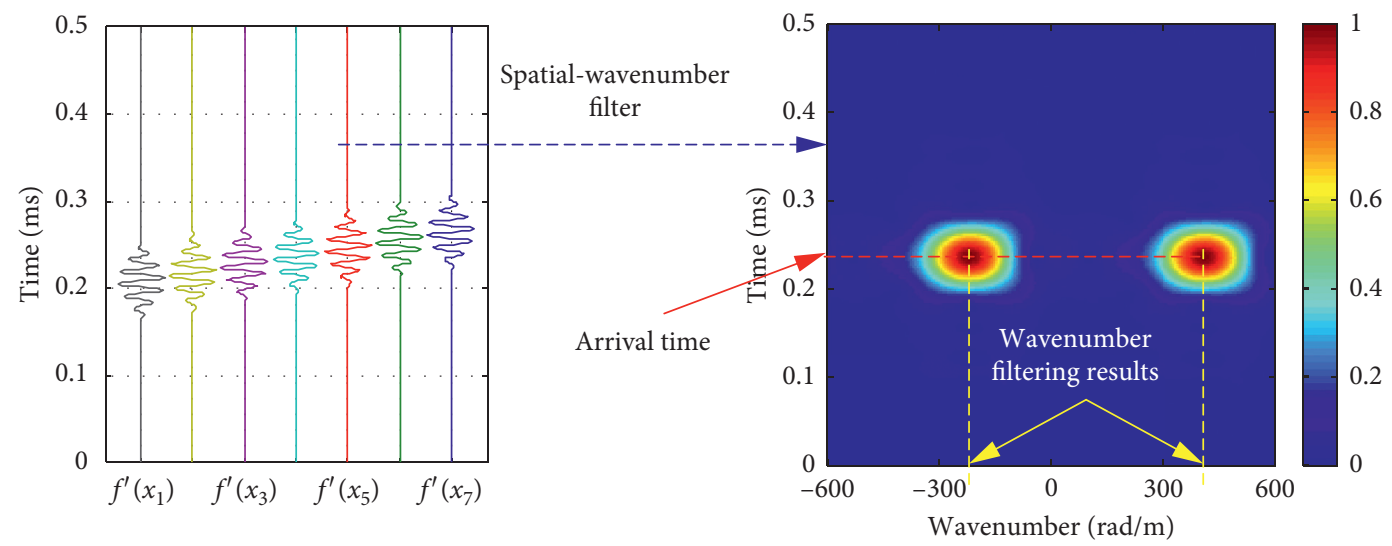

FIGURE 2: Example of a wavenumber-time image.

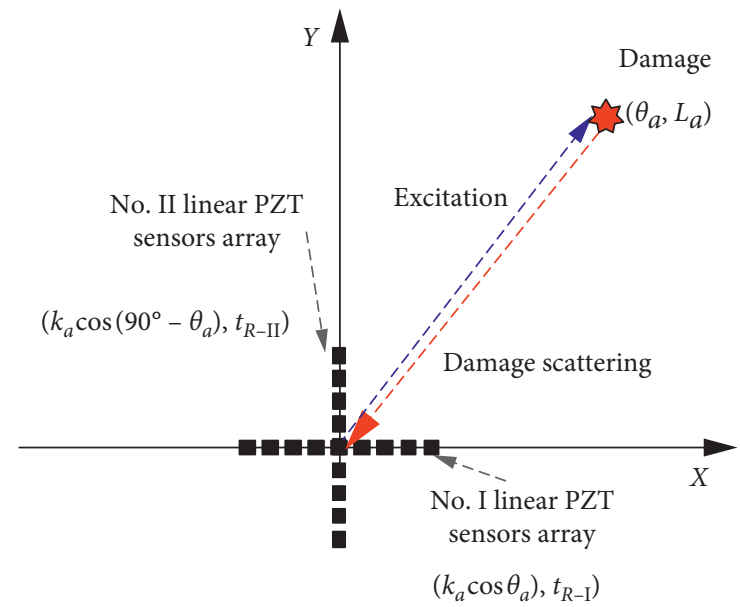

Figure 3: Schematic diagram of the damage localization.

\section{Validation of the Method}

4.1. Experimental Setup. The validation experimental system comprises an integrated SHM system, a cruciform PZT sensor array, and an epoxy laminate plate, as shown in Figure 4.

The dimensions of the epoxy laminate plate are $60 \mathrm{~cm} \times 60 \mathrm{~cm} \times 0.2 \mathrm{~cm} \quad$ (length $\times$ width $\times$ thickness). The epoxy laminate plate is stacked with 16 single layers, and the ply sequences are $\left[0_{2} / 90_{4} / 0_{2} / 0_{2} / 90_{4} / 0_{2}\right]$. The cruciform PZT sensor array is arranged in the middle of the lower part of the epoxy laminate plate. The two linear PZT sensor arrays of the cruciform PZT sensor array are numbered No.I and No.II. Each linear PZT sensor array consists of 7 PZT-5A sensors. The spatial sampling interval which is also the distance between the center points of two adjacent PZT sensors is $\Delta x=0.9 \mathrm{~cm}$. The PZT sensors in No.I PZT sensor array are labeled as PZT I-1,..., PZT I-7. The PZT sensors in No.II PZT sensor array are labeled as PZT II-1, ..., PZT II-7. A PZT sensor is pasted on the back of the specimen and the cross point of the cruciform PZT sensor array as the excitation element of the Lamb wave. The cross point of the cruciform PZT sensor array is $20 \mathrm{~cm}$ from the lower boundary of the epoxy laminate plate and $30 \mathrm{~cm}$ from the left and right boundaries of the epoxy laminate plate. The original point is set at the cross point of the cruciform PZT sensor array. In addition, the $X$ - and $Y$-axis of the Cartesian coordinates are set along the No.I PZT sensor array and No.II PZT sensor array, respectively. The Lamb wave velocity $c_{g}$ is measured by a PZT sensor pasted at the position of $90^{\circ}$ and $30 \mathrm{~cm}$, which is labeled as PZT 8. The integrated SHM system is developed by Professor Yuan research group [38].

In this experimental verification, the excitation signal was a modulated 5-peak narrowband signal [39]. The frequency and amplitude of the excitation signal are $50 \mathrm{kHz}$ and $\pm 70 \mathrm{~V}$. The sampling frequency and length of the Lamb wave are $10 \mathrm{MHz}$ and 8000 samples with 1000 presamples.

The experimental process is as follows: first, the Lamb wave velocity $c_{g}$ is measured using the Shannon wavelet transform [40]. The Lamb wave is excited by the excitation PZT sensor and propagates in the epoxy laminate plate. The corresponding Lamb wave signal is collected by PZT 8 . The excitation time and arrival time are calculated through the continuous complex Shannon wavelet transform. Then, the Lamb wave group velocity can be calculated as $c_{g}=1370 \mathrm{~m} / \mathrm{s}$ and applied to the following damage localization.

Second, the epoxy laminate plate is in the healthy status. The Lamb wave is excited by the excitation PZT sensor and propagates in the epoxy laminate plate. The corresponding Lamb wave signals collected by the cruciform PZT sensor array are the health reference signals $f_{\mathrm{HR}}$.

Third, six damages labeled $\mathrm{A}$ to $\mathrm{F}$ are applied to the epoxy laminate plate. Next, the corresponding Lamb wave signals collected by the cruciform PZT sensor array are the online monitoring signals $f_{\mathrm{OM}}$. The positions of these damages are shown in Figure 4(b) and Table 1.

4.2. Damage Localization. The damage $F$ is chosen as an example to validate in detail the proposed method and is located at $180^{\circ}$ and $20 \mathrm{~cm}$. First, the health reference signals $f_{\mathrm{HR}}$ are collected by the cruciform PZT sensor array, as shown in Figure 5.

After the damage $F$ is applied to the epoxy laminate plate, the online monitoring signals $f_{\mathrm{OM}}$ collected by the cruciform PZT sensor array are shown in Figure 6. 


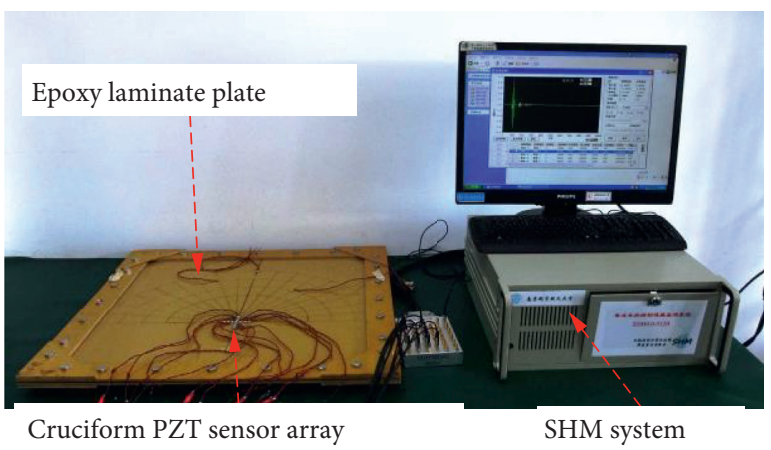

(a)

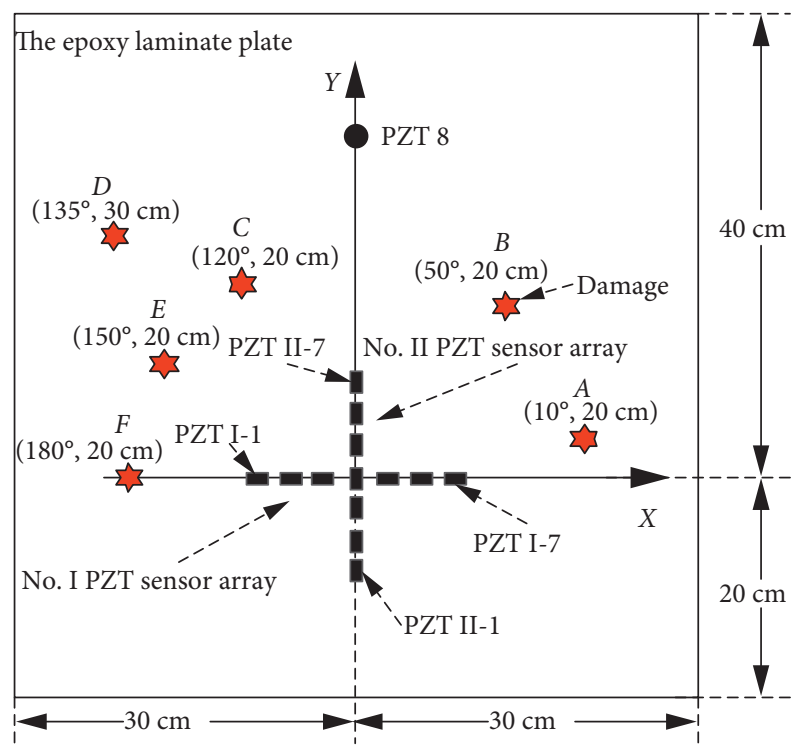

(b)

FIGURE 4: Experimental system for validating the damage localization based on the spatial-wavenumber filter. (a) Experiment setup. (b) Illustration of the cruciform PZT sensor array placement and damage positions.

TABLE 1: Damage localization results of the six damages.

\begin{tabular}{lcccccc}
\hline Damage label & $\left(k_{a-\mathrm{I}}, t_{R-\mathrm{I}}\right)(\mathrm{rad} / \mathrm{m}, \mathrm{ms})$ & $\begin{array}{c}\left(k_{a-\mathrm{II}}, t_{R-\mathrm{II}}\right) \\
(\mathrm{rad} / \mathrm{m}, \mathrm{ms})\end{array}$ & Start time $(\mathrm{ms})$ & $\begin{array}{c}\text { Localized position } \\
\left({ }^{\circ}, \mathrm{cm}\right)\end{array}$ & $\begin{array}{c}\text { Actual position } \\
\left({ }^{\circ}, \mathrm{cm}\right)\end{array}$ & $\begin{array}{c}\text { Damage localization } \\
\mathrm{error}(\mathrm{cm})\end{array}$ \\
\hline$A$ & $(382.0,0.3967)$ & $(58.6,0.3966)$ & 0.1031 & $(8.7,20.1)$ & $(10,20)$ & 0.5 \\
$B$ & $(244.6,0.3858)$ & $(309.5,0.3816)$ & 0.1031 & $(51.7,19.2)$ & $(50,20)$ & 1.0 \\
$C$ & $(-204.8,0.3991)$ & $(329.4,0.3991)$ & 0.1031 & $(121.9,20.3)$ & $(120,20)$ & 0.7 \\
$D$ & $(-286.3,0.5602)$ & $(278.4,0.5602)$ & 0.1031 & $(135.8,31.3)$ & $(135,30)$ & 1.4 \\
$E$ & $(-341.0,0.4237)$ & $(185.5,0.4189)$ & 0.1031 & $(151.5,21.8)$ & $(150,20)$ & 1.9 \\
$F$ & $(-387.9,0.4029)$ & $(3.9,0.3992)$ & 0.1031 & $(179.4,20.4)$ & $(180,20)$ & 0.5 \\
\hline
\end{tabular}

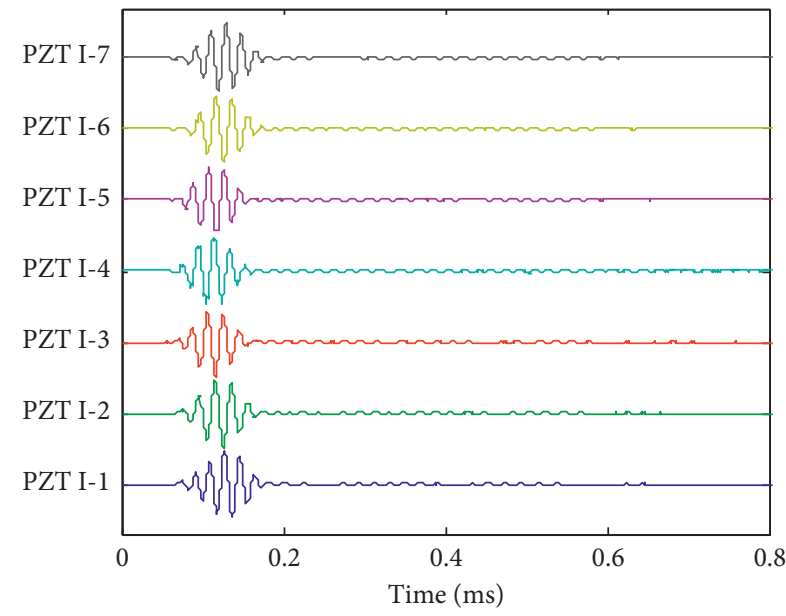

(a)

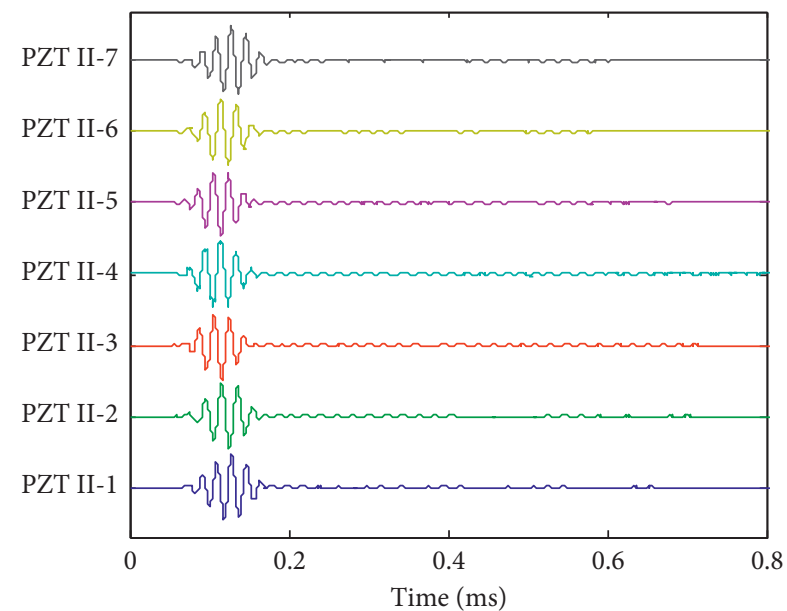

(b)

Figure 5: Health reference signals. (a) No.I PZT sensor array. (b) No.II PZT sensor array.

The damage scattering signals of damage $F$ can be extracted by subtracting the health reference signals $f_{\mathrm{HR}}$ from the online monitoring signals $f_{\mathrm{OM}}$, as shown in Figure 7.
According to the spatial sampling interval $\Delta x=0.9 \mathrm{~cm}$, the wavenumber filtering range was set to be from $-680 \mathrm{rad} /$ $\mathrm{m}$ to $680 \mathrm{rad} / \mathrm{m}$ with the wavenumber filtering interval $\Delta k=0.1 \mathrm{rad} / \mathrm{m}$. Then, the wavenumber-time images of 


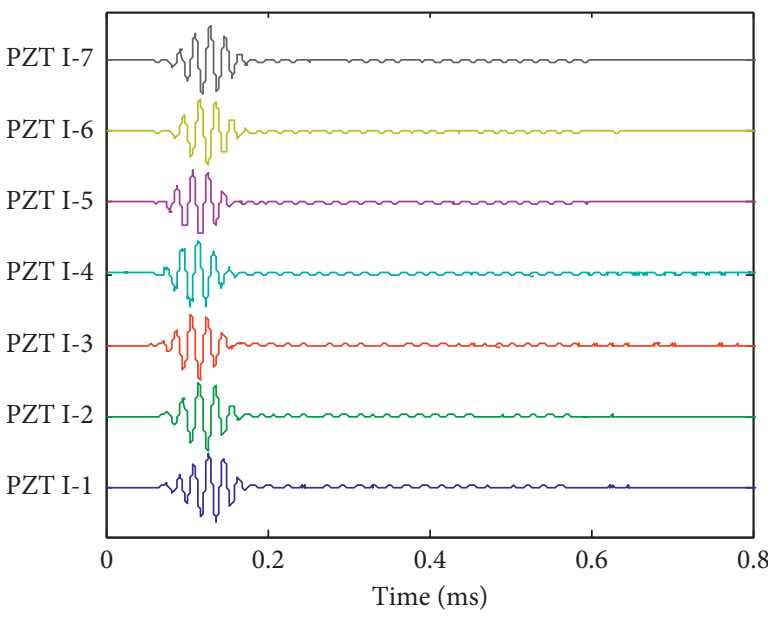

(a)

FIgURe 6: Online monitoring signals of the damage F. (a) No.I PZT sensor array. (b) No.II PZT sensor array.

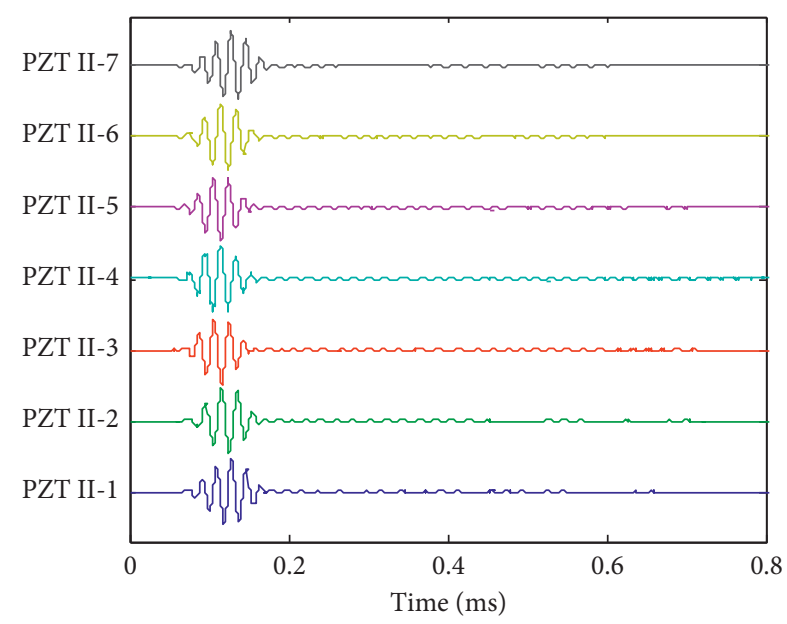

(b)

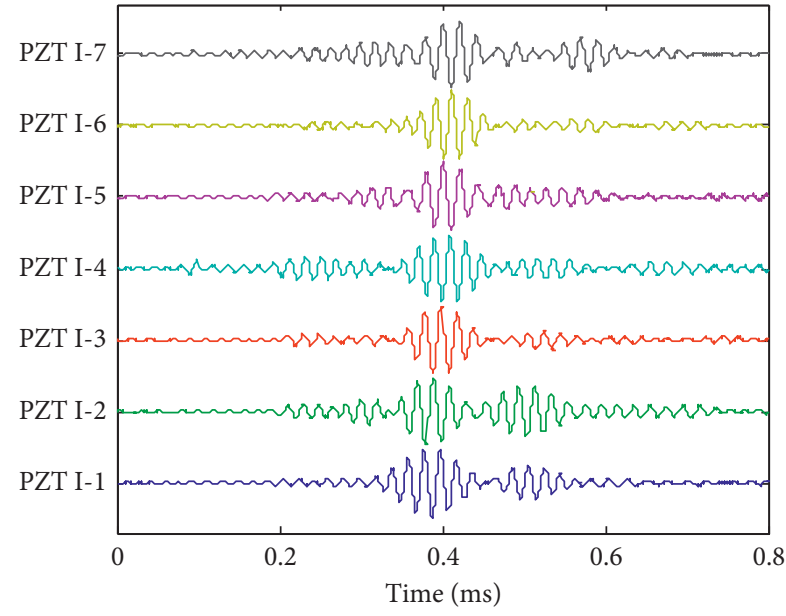

(a)

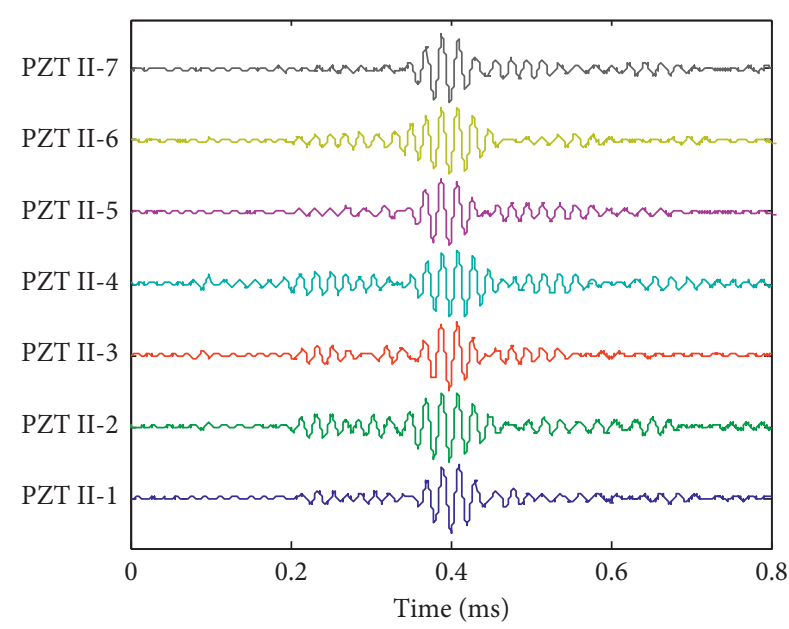

(b)

Figure 7: Damage scattering signals of the damage F. (a) No.I PZT sensor array. (b) No.II PZT sensor array.

damage $F$ can be obtained by spatial-wavenumber filtering of the damage scattering signals extracted from the online monitoring signals, as shown in Figure 8.

In Figure 8(a), two wavenumber filtering results $(-387.9 \mathrm{rad} / \mathrm{m}$ and $310.3 \mathrm{rad} / \mathrm{m})$ are shown that correspond to the point of the maximum value. Because there are two wavenumber filtering results of No.I PZT sensor array, the average arrival time difference $\left(t_{a}=0.0025 \mathrm{~ms}\right)$ between two adjacent sensors of No.I PZT sensor array can be calculated. Then, $k_{a-\mathrm{I}}=-387.9 \mathrm{rad} / \mathrm{m}$ is selected as the wavenumber of damage scattering signals collected by No.I PZT sensor array for $t_{a}=0.0025>0$. Furthermore, the arrival time of the damage scattering signals collected by No.I PZT sensor array is $t_{R-\mathrm{I}}=0.4029 \mathrm{~ms}$.

The wavenumber $\left(k_{a-\mathrm{II}}=3.9 \mathrm{rad} / \mathrm{m}\right)$ and arrival time $\left(t_{R-\mathrm{II}}=0.3992 \mathrm{~ms}\right)$ of the damage scattering signals collected by No.II PZT sensor array can also be obtained from Figure 8(b).

According to (18), the damage direction $\left(\theta_{a}=179.4^{\circ}\right)$ can be obtained using the wavenumbers $k_{a-\mathrm{I}}$ and $k_{a-\mathrm{II}}$, and the damage direction error is $-0.6^{\circ}$.

The excitation time $\left(t_{e}=0.1031 \mathrm{~ms}\right)$ of the Lamb wave is calculated by the continuous complex Shannon wavelet transform. Then, the distance $L_{a}=20.4 \mathrm{~cm}$ of the damage $F$ can be calculated by (19). Finally, the damage position $\left(179.4^{\circ}\right.$ and $\left.20.4 \mathrm{~cm}\right)$ is localized, and the damage localization error becomes $\Delta l=0.5 \mathrm{~cm}$.

According to the signal processing flow of damage $F$ discussed above, the six damage localization results and errors are listed in Table 1, and the damage localization image is shown in Figure 9. It can be seen from Table 1 that the $X$-axis projection wavenumbers of damages $A$ and $F$ 


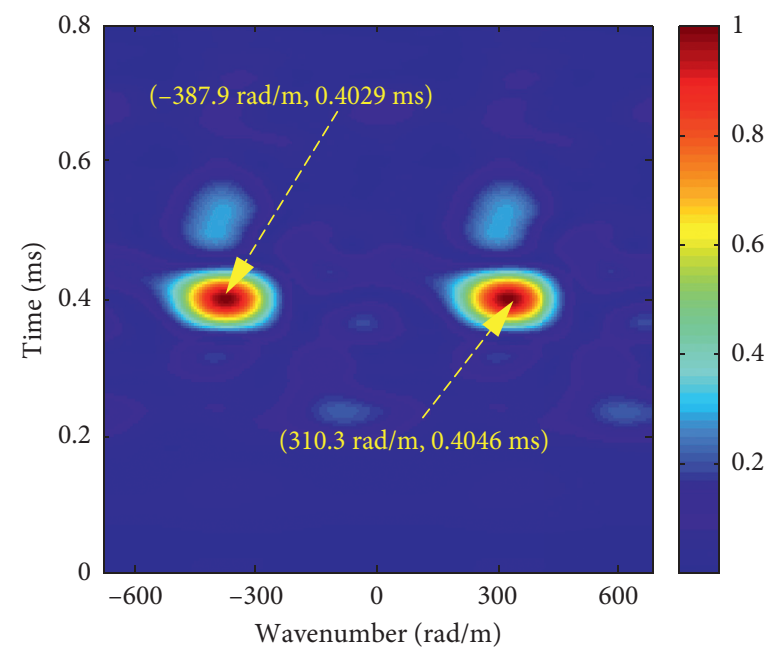

(a)

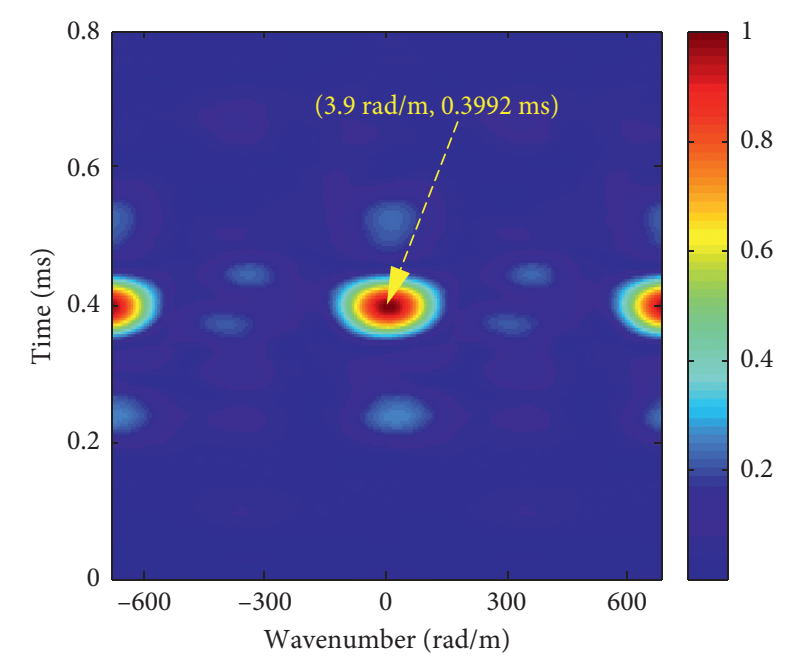

(b)

FIgURE 8: Wavenumber-time images of damage F. (a) No.I PZT sensor array. (b) No.II PZT sensor array.

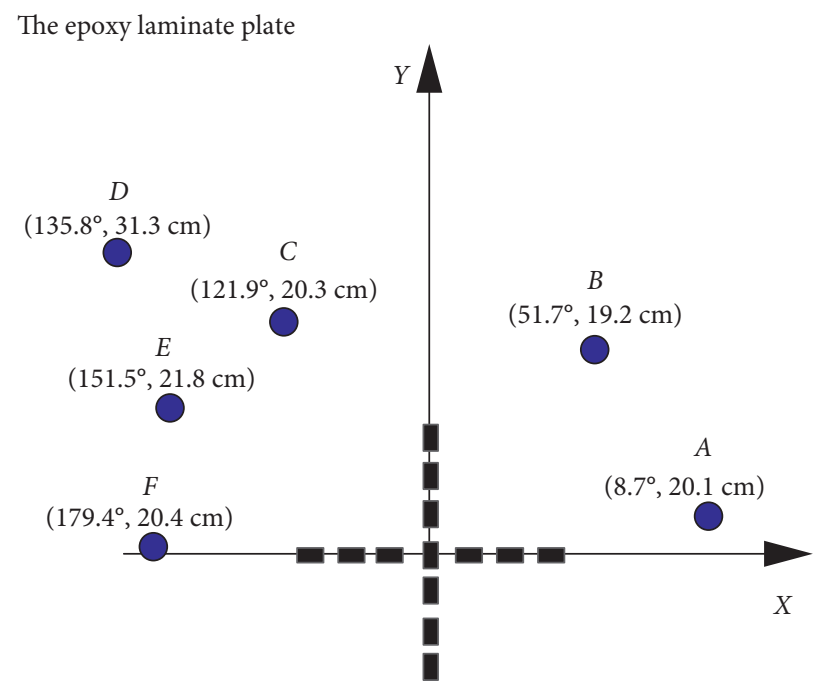

FIGURE 9: The damage localization image of the six damages.

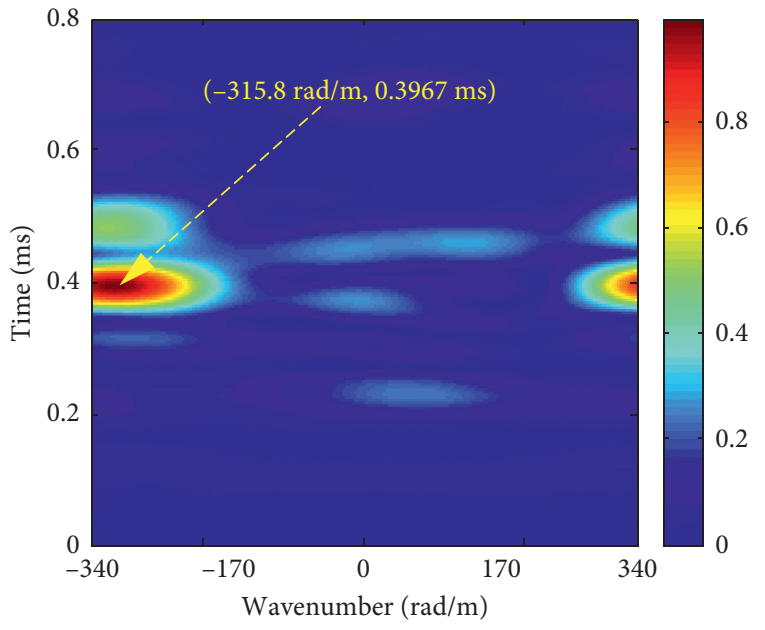

(a)

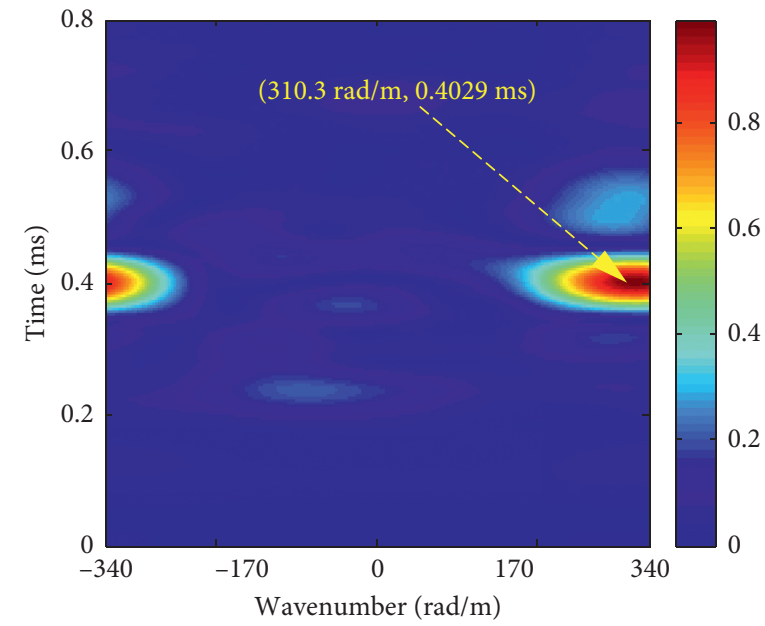

(b)

FIgURE 10: Wavenumber-time images of the conventional spatial-wavenumber filter method. (a) $k_{a-\text { I }}$ of damage $A$; (b) $k_{a-\text { I }}$ of damage $F$. 
exceed half of the spatial sampling wavenumber; the wavenumber-time images obtained by the conventional spatial-wavenumber filter method $[29,30]$, with the wavenumber filtering range from $-340 \mathrm{rad} / \mathrm{m}$ to $340 \mathrm{rad} / \mathrm{m}$, are shown in Figure 10. In Figure 10, the $X$-axis projection wavenumber of damage $A$ is $k_{a-\mathrm{I}}=-315.8 \mathrm{rad} / \mathrm{m}$, and the damage direction is $\theta_{a}=169.5^{\circ}$ with the damage direction error $159.5^{\circ}$. Similarly, the $X$-axis projection wavenumber of damage $F$ is $k_{a-\mathrm{I}}=310.3 \mathrm{rad} / \mathrm{m}$, and the damage direction is $\theta_{a}=0.7^{\circ}$ with the damage direction error $179.3^{\circ}$. It means that if the wavenumber of collected signal exceeds half of the spatial sampling wavenumber, the damage direction cannot be acquired correctly.

In the proposed method, the maximum filtering wavenumber is set to the spatial sampling wavenumber, and the two wavenumber filtering results are distinguished according to the average arrival time difference. The maximum damage localization errors are less than $2 \mathrm{~cm}$ in this experiment. The results indicate that the proposed method can improve the limitation of Nyquist-Shannon sampling theorem to the conventional spatial-wavenumber filter method, expand the filtering range of spatial-wavenumber filter to the spatial sampling wavenumber of the linear PZT sensor array, and thus expand the application of the spatialwavenumber filter based online damage monitoring.

\section{Conclusion}

In this paper, a frequency aliasing based spatial-wavenumber filter for online damage monitoring is proposed. In this method, the wavenumber filtering range of the spatialwavenumber filter is expanded to the spatial sampling wavenumber of the Lamb wave. Then, the wavenumber of the received Lamb wave signal is determined according to the average arrival time difference between two adjacent sensors in a linear PZT sensor array. The damage can be localized using this method and a cruciform PZT sensor array. We validated the results using an epoxy laminate plate, and the results show that the damage localization errors are less than $2 \mathrm{~cm}$. This method extends the wavenumber processing ability of the linear PZT sensor array using a software algorithm, without adding any hardware equipment. It is easily expanding the application of the spatial-wavenumber filter based online damage monitoring. However, depending on the group velocity of damage localization, the application of the proposed method may be limited; hence, further study is required. In addition, the influence of various factors on this method also needs to be studied further.

\section{Data Availability}

The data used to support the findings of this study are available from the corresponding author upon request.

\section{Conflicts of Interest}

The authors declare no conflicts of interest.

\section{Acknowledgments}

This work also benefited from the assistance of Professor Shenfang Yuan and Lei Qiu. This research was supported by the National Natural Science Foundation of China (no. 51705530), the Xuzhou Science and Technology Plan Project (no. KH17010), the Air Force Service Academy Youth Research Fund Project (no. KY2018D002A), and the 111 Project (no. D18003).

\section{References}

[1] S. Sony, S. Laventure, and A. Sadhu, "A literature review of next-generation smart sensing technology in structural health monitoring," Structural Control and Health Monitoring, vol. 26, Article ID e2321, 2019.

[2] M. Mitra and S. Gopalakrishnan, "Guided wave based structural health monitoring: a review," Smart Materials and Structures, vol. 25, Article ID 053001, 2016.

[3] S. Roy, P. Ladpli, and F.-K. Chang, "Load monitoring and compensation strategies for guided-waves based structural health monitoring using piezoelectric transducers," Journal of Sound and Vibration, vol. 351, pp. 206-220, 2015.

[4] P. Kudela, M. Radzienski, W. Ostachowicz, and Z. Yang, "Structural Health Monitoring system based on a concept of Lamb wave focusing by the piezoelectric array," Mechanical Systems and Signal Processing, vol. 108, pp. 21-32, 2018.

[5] X. Qing, W. Li, Y. Wang, and H. Sun, "Piezoelectric transducer-based structural health monitoring for aircraft applications," Sensors, vol. 19, no. 3, p. 545, 2019.

[6] G. Lu, Y. Li, T. Wang, H. Xiao, L. Huo, and G. Song, "A multidelay-and-sum imaging algorithm for damage detection using piezoceramic transducers," Journal of Intelligent Material Systems and Structures, vol. 28, no. 9, pp. 1150-1159, 2017.

[7] S. Shan, J. Qiu, C. Zhang, H. Ji, and L. Cheng, "Multi-damage localization on large complex structures through an extended delay-and-sum based method," Structural Health Monitoring: An International Journal, vol. 15, no. 1, pp. 50-64, 2016.

[8] Q. Xia, Y. Liu, Y. Lu, S. Cao, H. Zhang, and S. Ma, “A modified damage index probability imaging algorithm based on delayand-sum imaging for synthesizing time-reversed Lamb waves," Journal of Vibroengineering, vol. 21, pp. 2140-2147, 2019.

[9] Y. Ren, L. Qiu, S. Yuan, and F. Fang, "Gaussian mixture model and delay-and-sum based 4D imaging of damage in aircraft composite structures under time-varying conditions," $\mathrm{Me}$ chanical Systems and Signal Processing, vol. 135, Article ID 106390, 2020.

[10] T. Wang, S. Liu, J. Shao, and Y. Li, "Health monitoring of bolted joints using the time reversal method and piezoelectric transducers," Smart Materials and Structures, vol. 25, Article ID 025010, 2016.

[11] L. Zhang, C. Wang, L. Huo, and G. Song, "Health monitoring of cuplok scaffold joint connection using piezoceramic transducers and time reversal method," Smart Materials and Structures, vol. 25, Article ID 035010, 2016.

[12] A. Eremin, E. Glushkov, N. Glushkova, and R. Lammering, "Guided wave time-reversal imaging of macroscopic localized inhomogeneities in anisotropic composites," Structural Health Monitoring, vol. 18, no. 5-6, pp. 1803-1819, 2019.

[13] L. Zeng, J. Lin, and L. Huang, "A modified Lamb wave timereversal method for health monitoring of composite structures," Sensors, vol. 17, no. 5, p. 955, 2017. 
[14] B. Liu, T. Liu, Y. Lin, and J. Zhao, "An aircraft pallet damage monitoring method based on damage subarea identification and probability-based diagnostic imaging," Journal of Advanced Transportation, vol. 2019, Article ID 2568736, 12 pages, 2019.

[15] F. Li, H. Li, J. Qiu, and G. Meng, "Guided wave propagation in h-beam and probability-based damage localization: guided wave propagation in h-beam and damage localization," Structural Control and Health Monitoring, vol. 24, Article ID e1916, 2017.

[16] J. Zhao, X. Miao, F. Li, and H. Li, "Probabilistic diagnostic algorithm-based damage detection for plates with non-uniform sections using the improved weight function," Journal of Vibration Engineering \& Technologies, vol. 6, no. 3, pp. 249260, 2018.

[17] D. Li, Z. Jing, and M. Jin, "Plate-like structure damage location identification based on Lamb wave baseline-free probability imaging method," Advances in Mechanical Engineering, vol. 9, Article ID 168781401668570, 2017.

[18] Y. Sun, Y. Yuan, Q. Wang, L. Wang, E. Li, and L. Qiao, "Research on the signal reconstruction of the phased array structural health monitoring based using the basis pursuit algorithm," Computers, Materials \& Continua, vol. 58, no. 2, pp. 409-420, 2019.

[19] Z. Bai, S. Chen, Q. Xiao, L. Jia, Y. Zhao, and Z. Zeng, "Compressive sensing of phased array ultrasonic signal in defect detection: simulation study and experimental verification," Structural Health Monitoring, vol. 17, no. 3, pp. 434-449, 2018.

[20] O. Tofeldt and N. Ryden, "Lamb wave phase velocity imaging of concrete plates with 2D arrays," Journal of Nondestructive Evaluation, vol. 37, p. 4, 2018.

[21] Y. Ohara, K. Takahashi, Y. Ino, K. Yamanaka, T. Tsuji, and T. Mihara, "High-selectivity imaging of closed cracks in a coarse-grained stainless steel by nonlinear ultrasonic phased array," NDT \& E International, vol. 91, pp. 139-147, 2017.

[22] J. He and F.-G. Yuan, "Lamb wave-based subwavelength damage imaging using the DORT-MUSIC technique in metallic plates," Structural Health Monitoring: An International Journal, vol. 15, no. 1, pp. 65-80, 2016.

[23] Q. Bao, S. Yuan, F. Guo, and L. Qiu, "Transmitter beamforming and weighted image fusion-based multiple signal classification algorithm for corrosion monitoring," Structural Health Monitoring, vol. 18, no. 2, pp. 621-634, 2019.

[24] Y. Zhong, J. Xiang, X. Chen, Y. Jiang, and J. Pang, "Multiple signal classification-based impact localization in composite structures using optimized ensemble empirical mode decomposition," Applied Sciences, vol. 8, no. 9, p. 1447, 2018.

[25] T. Fu, Y. Wang, L. Qiu, and X. Tian, "Sector piezoelectric sensor array transmitter beamforming MUSIC algorithm based structure damage imaging method," Sensors, vol. 20, no. 5, p. 1265, 2020.

[26] H. Zuo, Z. Yang, C. Xu, S. Tian, and X. Chen, "Damage identification for plate-like structures using ultrasonic guided wave based on improved MUSIC method," Composite Structures, vol. 203, pp. 164-171, 2018.

[27] A. S. Purekar and D. J. Pines, "Damage detection in thin composite laminates using piezoelectric phased sensor arrays and guided lamb wave interrogation," Journal of Intelligent Material Systems and Structures, vol. 21, no. 10, pp. 995-1010, 2010.

[28] Y. Wang, S. Yuan, and L. Qiu, "Improved wavelet-based spatial filter of damage imaging method on composite structures," Chinese Journal of Aeronautics, vol. 24, no. 5, pp. 665-672, 2011.

[29] L. Qiu, B. Liu, S. Yuan, and Z. Su, "Impact imaging of aircraft composite structure based on a model-independent spatialwavenumber filter,” Ultrasonics, vol. 64, pp. 10-24, 2016.

[30] L. Qiu, B. Liu, S. Yuan, Z. Su, and Y. Ren, "A scanning spatialwavenumber filter and pzt 2-D cruciform array based on-line damage imaging method of composite structure," Sensors and Actuators A: Physical, vol. 248, pp. 62-72, 2016.

[31] Y. Ren, L. Qiu, S. Yuan, and Q. Bao, “On-Line multi-damage scanning spatial-wavenumber filter based imaging method for aircraft composite structure,” Materials, vol. 10, p. 519, 2017.

[32] Y. Ren, L. Qiu, S. Yuan, and Z. Su, "A diagnostic imaging approach for online characterization of multi-impact in aircraft composite structures based on a scanning spatialwavenumber filter of guided wave," Mechanical Systems and Signal Processing, vol. 90, pp. 44-63, 2017.

[33] G. Fan, H. Zhang, H. Zhang, W. Zhu, and X. Chai, "Lamb wave local wavenumber approach for characterizing flat bottom defects in an isotropic thin plate," Applied Sciences, vol. 8, no. 9, p. 1600, 2018.

[34] Z. Tian and L. Yu, "Lamb wave frequency-wavenumber analysis and decomposition," Journal of Intelligent Material Systems and Structures, vol. 25, no. 9, pp. 1107-1123, 2014.

[35] P. Kudela, M. Radzieński, and W. Ostachowicz, "Identification of cracks in thin-walled structures by means of wavenumber filtering," Mechanical Systems and Signal Processing, vol. 50-51, pp. 456-466, 2015.

[36] J. Cai, S. Yuan, X. P. Qing, F.-K. Chang, L. Shi, and L. Qiu, "Linearly dispersive signal construction of Lamb waves with measured relative wavenumber curves," Sensors and Actuators A: Physical, vol. 221, pp. 41-52, 2015.

[37] V. Memmolo, E. Monaco, N. D. Boffa, L. Maio, and F. Ricci, "Guided wave propagation and scattering for structural health monitoring of stiffened composites," Composite Structures, vol. 184, pp. 568-580, 2018.

[38] L. Qiu and S. Yuan, "On development of a multi-channel PZT array scanning system and its evaluating application on UAV wing box," Sensors and Actuators A: Physical, vol. 151, no. 2, pp. 220-230, 2009.

[39] G. Zhang, W. Gao, G. Song, and Y. Song, "An imaging algorithm for damage detection with dispersion compensation using piezoceramic induced lamb waves," Smart Materials and Structures, vol. 26, Article ID 025017, 2017.

[40] L. Qiu, S. Yuan, X. Zhang, and Y. Wang, "A time reversal focusing based impact imaging method and its evaluation on complex composite structures," Smart Materials and Structures, vol. 20, no. 10, Article ID 105014, 2011. 